\title{
Surgical management of essential blepharospasm
}

\author{
A K Bates, B L Halliday, C S Bailey, J R O Collin, A C Bird
}

\begin{abstract}
We have reviewed the surgical management of essential blepharospasm over the last 15 years, comparing the results from facial nerve avulsion with those from orbicularis muscle stripping. After facial nerve avulsion $\mathbf{5 0 \%}$ of patients remained free of troublesome spasm for 15 months after surgery, but only $25 \%$ remained so for more than two years. Following orbicularis oculi myectomy $50 \%$ of patients were free of troublesome spasms for 30 months after surgery and $55 \%$ of patients had relief from spasm for more than two years. Secondary effects of the two procedures are compared and are found to be fewer after orbicularis myectomy. There were no major complications after either form of surgery. Botulinum toxin is the treatment of first choice for this condition. If this becomes ineffective or inconvenient, surgical treatment is warranted and should not be deferred for fear of severe side effects of treatment, since these are rare. Protractor myectomy gives longer relief from blepharospasm than facial nerve avulsion and has fewer complications. However, it is technically difficult, time consuming, and has greater peroperative morbidity. Facial nerve avulsion may therefore still have a role in selected patients.
\end{abstract}

Essential blepharospasm is an idiopathic disorder of progressive involuntary spasms of the orbicularis oculi and upper facial muscles. It may occur in isolation or as part of a spectrum of dystonic movements of other facial, oral, mandibular, or cervical muscles. The condition usually presents in middle age and is more common in women than men. ${ }^{1}$ It often begins with episodes of increased blinking of variable duration. This gradually progresses to involuntary spasms of eyelid closure. The course thereafter is very variable, with remissions and exacerbations and in some cases progression to involvement of other muscle groups. Eventually the patients may develop such severe spasms that they become functionally blind and cannot work or care for themselves. ${ }^{2}$

Spasms may be precipitated by certain factors such as reading, driving, stress, or bright light, and they may be alleviated by others such as talking, walking, or relaxation. ${ }^{2}$

A wide variety of treatments have been used in an attempt to alleviate blepharospasm. ${ }^{12}$ Psychotherapy, hypnosis, acupuncture, faith healing, and herbal remedies have all been tried, with limited success. Several drugs have been reported to ameliorate specific types of blepharospasm, but their efficacy seems to be limited and temporary in most cases. ${ }^{1}$ Drugs which have been used include antidepressants, antianxiety agents, anticonvulsants, antiParkinsonian drugs, and muscle relaxants.

Botulinum-A toxin is currently regarded as the best temporary treatment for blepharospasm. ${ }^{34}$ By interference with acetylcholine release from nerve terminals it results in temporary paralysis of the injected muscles. Subcutaneous injections of botulinum toxin into the upper and lower lid and eyebrow give temporary relief of eyelid spasm and initially have a high degree of patient acceptance. Repeat injections are required initially after three-four months, and then more frequent injections of larger doses may become necessary to control spasms. $^{5}$

Many surgical procedures have been tried to interrupt the pathway from the motor cortex to the orbicularis oculi muscles. 'Initially they took the form of peripheral facial neurectomy by alcohol injections, surgical sectioning, or percutaneous thermolysis. ${ }^{6-9}$ With time the most popular surgical technique became selective peripheral facial nerve avulsion. ${ }^{11}$

More recently, however, a direct approach to the muscle has been advocated by stripping of the orbicularis and the brow muscle fibres combined with brow fixation. ${ }^{12-14}$ At the present time both selective facial nerve avulsion and orbicularis myectomy are performed at Moorfields Eye Hospital for patients who require surgical treatment.

We have reviewed the management of patients in this hospital with essential blepharospasm over the past 15 years, during which time botulinum toxin has become established as the initial treatment for this condition. We have looked at the long-term success and complications of facial nerve avulsion and compared them with the results from the newer muscle stripping procedure. We have then attempted to draw some conclusions as to the role of each of these procedures in the management of essential blepharospasm.

\section{Patients and methods}

We reviewed the case notes of 56 patients who received surgical treatment for essential blepharospasm between 1975 and 1990 at Moorfields Eye Hospital. Facial nerve avulsion has been performed throughout this period; orbicularis myectomy was first performed in 1983.

The following information was recorded: treatment received prior to surgery, type of surgery, duration of relief from spasms after surgery, complications or secondary effects of surgery, and further treatment necessary after surgery to obtain relief from blepharospasm.

Facial nerve avulsion was performed under general anaesthesia without muscle relaxation.
Correspondence to:

Mr A K Bates, FRCS

Accepted for publication

11 January 1991
City Road, London

A K Bates

B L Halliday

I S O O Collin

J R O Collin 
The facial nerve was approached at the anterior border of the parotid gland. All temporal, zygomatic, and buccal branches were identified by nerve stimulation and avulsed, as were any branches of the mandibular branch which were found to cause contraction of the muscles of the upper face.

Protractor myectomy was performed under general anaesthesia. In the first two cases all four lids were operated on. But because this surgery took more than four hours and involved consider able blood loss subsequent patients had only upper lid surgery in the first instance followed by lower lid surgery at a later stage if blepharospasm was not adequately controlled.

The excision of the upper eyelid orbicularis fibres and brow protractor muscles (corrugator supraciliaris and procerus) was carried out through a brow and eyelid incision. All visible muscle fibres were removed, while the surgeon attempted to preserve the supraorbital nerve. The levator aponeurosis was reinserted and secured to the tarsus to correct any ptosis, and the brows were elevated and fixed to periosteum with a series of non-absorbable sutures.

Even upper lid surgery alone is time consuming, taking up to three hours, and may result in major blood loss. On two occasions it was necessary to defer surgery on the second side until the patient was haemodynamically stable.

\section{Results}

Surgical treatment for essential blepharospasm was performed in 56 patients. As their primary surgical procedure 48 underwent facial nerve avulsion and eight orbicularis stripping. A further three patients then went on to have orbicularis stripping after facial nerve avulsion, making 11 in total.

Of the 48 patients undergoing facial nerve avulsion 28 were female, 20 male, with mean age at surgery of $61 \cdot 2$ years (range 36-83 years). Of these patients 35 had tried medications for their blepharospasm prior to surgery with no longterm success in any case. Fourteen had been controlled for periods of up to $17 \cdot 5$ months with botulinum toxin therapy, and one had had bilateral blepharoplasty previously.

After facial nerve avulsion the mean follow-up was 43.4 months (range 1-154). In 15 of the 48 patients (31\%) blepharospasm was controlled to an acceptable level throughout follow-up after one operation. Another six were controlled following reavulsion of residual nerve fibres in the early stages after surgery or of regenerating nerve fibres at a later stage (in total 14 patients underwent repeat facial nerve avulsion, four within four months of the initial procedure and 10 later-mean 14.4 months, range 1-34 months). In $27(56 \%)$ patients blepharospasm was not adequately controlled by one or more facial nerve avulsions during the period of follow-up. Of these, 19 went on to have botulinum toxin therapy, five had medical therapy, and three had the orbicularis stripping procedure.

Twenty (42\%) patients required one or more corrective lid procedures following facial nerve avulsion - 16 for ectropion, four for brow ptosis, and two for dermatochalasis. No patients suffered from significant long-term exposure keratitis, though all patients were routinely given tear supplementation in the early postoperative period. Epiphora was a common complaint soon after surgery but settled in the majority of cases, and in no case was lacrimal surgery required. Five patients were troubled by collections of saliva in the parotid gland, and in three cases this necessitated multiple aspirations over a few days until it settled.

Of the 11 patients undergoing the orbicularis stripping procedure nine were female and two male, and their mean age was 64.4 years (range 51-78 years). Prior to surgery seven of the patients had undergone medical therapy. Six had been controlled with botulinum toxin injections for a mean period of 17 months until the injections were no longer effective or were required at too frequent intervals to be practical. Three had troublesome blepharospasm despite previous facial nerve avulsion. After protractor myectomy the mean follow-up was $23 \cdot 7$ months (range 1-80 months).

Eight $(72 \%)$ of the 11 patients had acceptable control of blepharospasm throughout follow-up after the upper lid orbicularis stripping procedure alone. Three (27\%) patients required further treatment for blepharospasm. This consisted of lower lid orbicularis stripping alone in one case, lower lid orbicularis stripping followed by botulinum toxin injections in the second case, and botulinum toxin injections alone in the third case.

After the orbicularis stripping procedure most of the patients had marked postoperative swelling and bruising, but this settled. Only one patient required corrective lid surgery for a lower lid ectropion, which followed lower lid orbicularis stripping. Three patients were treated for minor degrees of corneal exposure but only for a short time after surgery. One patient, who was known to have partial nasolacrimal duct obstruction prior to surgery, now has persistent epiphora thought to be due to the additional effect of reduced function of the orbicularis mediated lacrimal pump and is awaiting dacryocystorhinostomy.

The probability of recurrence of blepharospasm with time after facial nerve avulsion and orbicularis myectomy was plotted after KaplanMeier analysis (Fig 1).

\section{Discussion}

At the present time 150 patients with essential blepharospasm are controlled by botulinum toxin injections at Moorfields Eye Hospital. Of these, 32 also require supplemental medical therapy, mostly with benzhexol. The mean follow-up time for these patients is $\mathbf{4 0}$ months. Patients who respond poorly to botulinum toxin injections either initially or after repeated injections are candidates for surgical treatment. Since botulinum toxin therapy became widely available in 1985 a total of 20 patients have undergone surgical treatment for blepharospasm after failure of toxin treatment. In the 5-year period prior to this 25 patients underwent surgical treatment. It is our belief that the number of patients requiring surgical treatment for blepharospasm has 
declined since botulinum toxin became available, but it is too early yet to say what proportion of those patients at present controlled with toxin will ultimately require surgical intervention.

It is very difficult to make an objective comparison of the relative success of different treatments for blepharospasm in terms of actual reduction of spasms. ${ }^{12}{ }^{14}$ However, an important measure is the proportion of patients who require further treatment to control their blepharospasm while under review. After facial nerve avulsion 15 of the 48 (31\%) patients required no further treatment and a further six were controlled after a second avulsion procedure. Thus $43.75 \%$ in all were controlled with facial nerve surgery alone throughout the study. After orbicularis myectomy eight of the 11 patients were relieved by a single operation (73\%) and one other after lower lid stripping, making $81.8 \%$ in all controlled by orbicularis muscle surgery throughout the period of review. This is similar to the findings of other series. ${ }^{1314}$ However, since follow-up is much longer in the facial nerve avulsion group, and therefore recurrence more likely, Kaplan-Meier analysis is necessary to provide a more accurate comparison of the two groups (Fig 1). After facial nerve avulsion $50 \%$ of patients had recurrence of disabling spasms within 15 months of surgery and only $25 \%$ had relief from spasms for more than two years. Other series have quoted recurrence rates of $35-40 \%$ at 15 months and 50 $55 \%$ at two years, ${ }^{7}$ but this obviously depends on a highly subjective assessment by the patients.

After orbicularis myectomy relief is more prolonged, with a $50 \%$ recurrence rate not observed until almost 30 months after surgery and $55 \%$ of patients obtaining relief for more than two years. Myectomy appears to offer the longer relief, but both methods require adjunctive treatment to maintain relief from blepharospasm.

It is important to consider how successful each of these treatments was in combination with adjunctive therapy, medications, or toxin rather than in isolation. It is well known that such

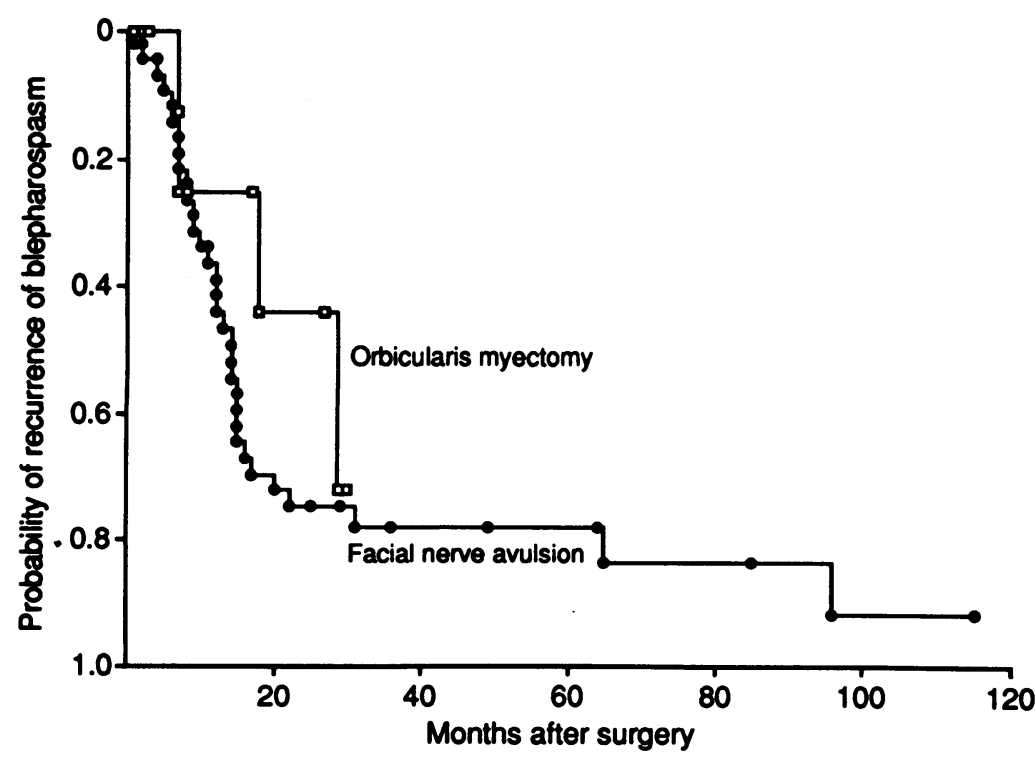

Figure 1 Kaplan-Meier curve for probability of recurrence of blepharospasm after surgery. therapy is more successful after either facial nerve avulsion or myectomy. ${ }^{15}$ All the patients who have required further treatment with botulinum toxin after myectomy have had a good response, and their spasms are well controlled. Following facial nerve avulsion this has also been true with the exception of three $(6 \%)$ patients whose spasms were not well controlled and so underwent myectomy with good effect.

Secondary effects of facial nerve avulsion that may require surgery include paralytic ectropion, epiphora, lagophthalmos, exacerbation of upper lid dermatochalasis, lip paresis, drooping of the mouth, collection of saliva, and a variable degree of loss of facial expression. ${ }^{813}$

A change in facial appearance is inevitable after this form of surgery, with drooping of the brows and of the corners of the mouth. However, after bilateral surgery the appearance of the face is symmetrical and fairly acceptable cosmetically. The loss of facial expression is obviously a serious consequence but one which was considered to be preferable to disabling blepharospasm by all the patients in this series, as has been the case in other series. ${ }^{11}$

Secondary effects of myectomy are reported to be less than those of facial nerve avulsion, ${ }^{13}$ and we found this to be the case. Those commonly described include considerable swelling and ecchymosis postoperatively, numbness of the forehead due to supraorbital nerve damage, chronic periorbital lymphoedema, exposure keratitis, and ectropion.

When the complications of the two forms of surgery are compared, myectomy appears to be the safer procedure. Only one of the 11 patients required surgical treatment for complications of myectomy (ectropion repair), whereas 20 of the 48 patients who underwent facial nerve avulsion required further surgical treatment for ectropion, brow or lid ptosis. This is similar to the findings of other series. ${ }^{8}$ In neither group did any patient suffer from severe corneal exposure, and though watering of the eyes was a common complaint in both groups only one patient was troubled sufficiently to consider lacrimal surgery.

Both groups of patients were of similar age and sex, and had similar treatment for blepharospasm prior to surgery. The decision as to whether myectomy or facial nerve avulsion was performed depended simply on which consultant they were referred to. In this series myectomy offered more prolonged relief from blepharospasm than facial nerve avulsion. Furthermore myectomy had fewer secondary effects, though no patient in either group suffered from severe corneal exposure or other major complication. The major disadvantage of myectomy is that it is a technically difficult and time consuming procedure, with the possibility of major blood loss. ${ }^{1213}$

In conclusion, we believe that botulinum toxin is the treatment of first choice in essential blepharospasm. If symptomatic spasms recur, surgery is justified and should not be deferred, as serious complications appear to be rare after either nerve avulsion or myectomy. In otherwise fit and healthy patients myectomy is the surgical treatment of first choice, because of its longer effect and fewer complications. However, facial 
nerve avulsion may still have a role as a primary procedure in the elderly or infirm because of its lower peroperative morbidity.

1 Henderson JW. Essential blepharospasm. Trans Am Ophthalmol 1956; 54: 453-20.

2 Bird AC, McDonald WI. Essential blepharospasm. Trans Ophthalmol Soc UK 1975; 95: 250-3.

3 Scott AB, Kennedy RA, Stubbs HA. Botulinum-A toxin injection as a treatment for blepharospasm. Arch Ophthalmol 1985; 103: 347-50.

4 Arthurs B. Treatment of blepharospasm with medication surgery, and type A botulinum toxin. Can $\mathcal{f}$ Ophthalmol 1987; 22: 24-8.

5 Jordan DR, Patrinely JR, Anderson RL. Thiese SM. Essential blepharospasm and related dystonias. Surv Ophthalmol 1989; 34: 123-32.

6 Callahan A. Blepharospasm with resection of part of orbicularis nerve supply. Arch Ophthalmol 1963; 70: 508-11.

7 Callahan A Surgical correction of intractable blepharospasm. Technical improvements. Am $\mathcal{f}$ Ophthalmol 1965; 60: 788-
8 Frueh BR, Callahan A, Dortzbach RK, et al. The effects of differential section of the VII nerve on patients with intractable blepharospasm. Trans Am Acad Ophthalmol 1976; 81: 595-602.

9 Dorzbach RK. Complications and surgery for blepharospasm. Am f Ophthalmol 1973; 75: 142-7.

10 Reynolds DH. Differential section of the facial nerve for blepharospasm. In: Smith JLS, eds. Neuro-ophthalmology. St Louis:

11 Reynolds DH, Smith JL, Walsh TJ. Differential section of the facial nerve for blepharospasm. Trans Am Acad Ophthalmol 1967; 71: 656-64.

12 Gillium WN, Anderson RL. Blepharospasm surgery: an anatomic approach Arch Ophthalmol 1981; 99: 1056-62.

13 McCord CD, Coles WH, Shore JW, Spector R, Putnam JR. Treatment of essential blepharospasm. I Comparison of facial nerve avulsion and eyebrow-eyelid muscle stripping procedure. Arch Ophthalmol 1984; 102: 266-8.

14 Jones TW, Waller RR, Samples JR. Myectomy for essential blepharospasm. Mayo Clin Proc 1985; 60: 663-6.

15 Shore JW, Leone CR, O'Connor PS, et al. Botulinum toxin for the treatment of essential blepharospasm. Ophthalmic Surg $1986 ; 17: 747-53$ 